\title{
A Large Prime Gap
}

\section{By Sol Weintraub}

\begin{abstract}
A prime gap of 654 (653 consecutive composites) is found near $1.1 \times 10^{16}$.
\end{abstract}
A prime gap of 654 was found following the prime 11000001446613353 . The highest gap previously published, 652, follows the prime 2614941710559 , (Brent [1]). Only smaller gaps occur below that prime. Theoretically, a gap of magnitude at least $n$ can be found following the integer $n !+1$ but this is of little practical value. For example, $654 ! \sim 1.47 \times 10^{1559}$.

Only four other gaps exceeding 500 were found in the interval between $N=1.1$ $\times 10^{16}$ and $N+1.5 \times 10^{9}$. According to Shanks' [2] conjecture, a gap of approximate size 1364 should exist somewhere below this $N$; but gaps of that size are very rare.

The five gaps exceeding 500 in this interval are

$\begin{array}{cc}\text { GAP } & \text { FOLLOWING } \\ 546 & 11000000002331551 \\ 510 & 11000000370769591 \\ 528 & 11000000410410853 \\ 504 & 11000000593773869 \\ 654 & 11000001446613353\end{array}$

Listed below are the maximum gaps in each interval of 100 million, beginning at $N=1.1 \times 10^{16}$ and ending at $N+1.5 \times 10^{9}$ :

$546,468,484,510,528,504,494,484,460,486,486,496,496,476,654$

e.g., the first number, 546, is the maximum gap between $N=1.1 \times 10^{16}$ and $N+10^{8}$.

For purposes of comparison, the maximum gaps (per 100 million) beginning at $1.1 \times 10^{13}$ are:

428, 396, 388, 370, 438, 356, 492, 380, 394, 390, 440, 396, 418, 384, 392.

Again, this shows that the occurrence of large prime gaps is exceedingly rare.

Department of Mathematics

Queens College, CUNY

Flushing, New York 11367

1. R. Brent, "The first occurrence of large gaps between successive primes," Math. Comp., v. 27, 1973, pp. 959-963.

2. D. Shanks, "On maximal gaps between successive primes," Math. Comp., v. 18, 1964, pp. 646-651.

Received February 21, 1980; revised July 7, 1980.

1980 Mathematics Subject Classification. Primary 10A20, 10A25, 10A40. 